Amplification and Compressor Technology for "Split Beam", High Energy Short Pulse Generation

R.J. Beach, V.K. Kanz, W.J. Clark, C.P.J. Barty, M.C. Rushford, J. Hernandez, G. Tietbohl

January 31, 2008 
This document was prepared as an account of work sponsored by an agency of the United States government. Neither the United States government nor Lawrence Livermore National Security, LLC, nor any of their employees makes any warranty, expressed or implied, or assumes any legal liability or responsibility for the accuracy, completeness, or usefulness of any information, apparatus, product, or process disclosed, or represents that its use would not infringe privately owned rights. Reference herein to any specific commercial product, process, or service by trade name, trademark, manufacturer, or otherwise does not necessarily constitute or imply its endorsement, recommendation, or favoring by the United States government or Lawrence Livermore National Security, LLC. The views and opinions of authors expressed herein do not necessarily state or reflect those of the United States government or Lawrence Livermore National Security, LLC, and shall not be used for advertising or product endorsement purposes.

This work performed under the auspices of the U.S. Department of Energy by Lawrence Livermore National Laboratory under Contract DE-AC52-07NA27344. 


\title{
Amplification and Compressor Technology for "Split Beam", High Energy Short Pulse Generation
}

\author{
LDRD Project Tracking Code: 05-ERD-062 \\ Ray Beach, Principal Investigator
}

\begin{abstract}
High energy density science and inertial fusion are of strategic importance for national security. Existing and planned facilities dedicated to such research could be significantly enhanced by the addition of a high-power short-pulse laser. Our research has provided part of the critical enabling R\&D that would allow such enhancements. Investigations included the R\&D issues related to creating an integrated system for the injection, setup, compression, and monitoring of high-energy, split-beam, short pulse generation from a single aperture of a Nd:glass laser system. In the course of our R\&D new amplifier and pulse compressor technologies were developed that meet the stringent operating requirements and architectural constraints of large-scale amplification systems that are capable of producing $\mathrm{kJ}$-class pulses.
\end{abstract}

\section{Introduction/Background}

The generation of multiple, kilo joule-class, picosecond pulses from a single aperture of a modern Nd:glass laser system has never been accomplished. Production of such short pulses requires new amplifier and pulse compressor technologies that are compatible with the stringent operating requirements and architectural constraints of large-scale amplifications systems and that are capable of producing kJ-class, picosecond pulses in a vacuum environment without component damage. The concepts of split beam amplification and compact mixed grating pulse compressors were studied and submitted as new inventions as part of the recent high energy short pulse strategic initiative. ${ }^{1,2}$ In the first case, it was suggested that two or more, separately timed short pulses could be generated from a single aperture of a large-scale laser amplifier chain by injecting two or more spatially separated, sub-aperture, chirped pulses into the amplifier. By doing so it may be possible to generate multiple time-frame $\mathrm{x}$-ray radiographs of laser driven experiments. In the second instance, it was suggested that a new arrangements of diffraction gratings could reduce by $500 \%$ the footprint of chirped pulse, pulse compressors. These compact arrangements are now being considered for deployment at several labs around the world. While the conceptual layout of a split-beam, compact, mixed grating pulse compressor has been considered, the numerous issues related to a real system have never been studied in detail. This ER investigated for the first time the R\&D issues related to injection, setup, compression and monitoring of high energy, splitbeam, short pulse generation. In particular it will investigate for the first time the R\&D issues associated with an integrated system:

a) new techniques for multiple, sub-aperture pulse generation, timing and pulse width control 
b) new methods for precision pointing and characterization of injected, split beam pulses

c) new methods for precision alignment of a folded, mixed grating pulse compressor

d) new methods for precision alignment of split beam pulse compressors

e) new methods for characterization of compressed spilt beam pulse alignment

f) evaluation of cross talk between split beam sub-apertures in a folded relay imaged optical amplifier, mixed grating pulse compressor

g) evaluation of the effects of vacuum isolation on optic alignment and beam collimation

\section{Research Activities}

Our research focused on technologies appropriate to applications of interest to DOE. We investigated the techniques and technology components to enable the amplification of two or more beams simultaneously in a single amplifier chain. This concept of split amplification, in which spatially and temporally independent beams are sent through a single amplifier aperture is novel to LLNL and has not been demonstrated elsewhere. Risk issues that needed be addressed for this proposed technique to be potentially applicable to the generation of multiple time-frame x-ray radiographs of laser driven experiments include: precision parallelism of the input sub-aperture beams and the possibility of cross talk between beams. In the case of cross talk between the early pulse and the trailing pulse, there could result a prepulse structure which would perturb the trailing pulse target in a multiframe backlighting experiment. Cross talk between beams can arise at the injection plane of the split beams into the amplifier system, at the transport spatial filters in the amplifier chain and in the folded, mixed grating pulse compressor. The technical approach followed used two spatially separated sub apertures as shown in Fig. 1

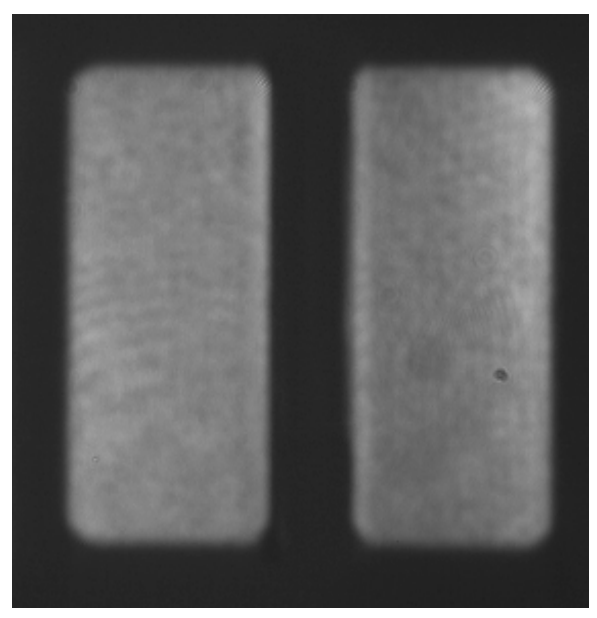

Fig. 1. Figure shows our technical approach in which two spatially separated sub-apertures are injected into the amplifier chain and then compressed with separate folded, mixed grating pulse compressors. 
In addition to the development of the technological approach for precision pointing of the split beams, we also investigated an automatic alignment system capable of handling a split beam configuration.

Figure 2 shows an example of a compact mixed grating compressor design for a single beam, which consists of four separate gratings. Also shown in Fig. 2 is the pathway for a single beam through the compressor. The beams come in through the top of the compressor as shown in Fig. 2 and reflect from mirror AM4 to follow the horizontal beam path shown. After diffracting from G4, mirror AM5 sends the beam out of the compressor tank to the target chamber center (TCC).

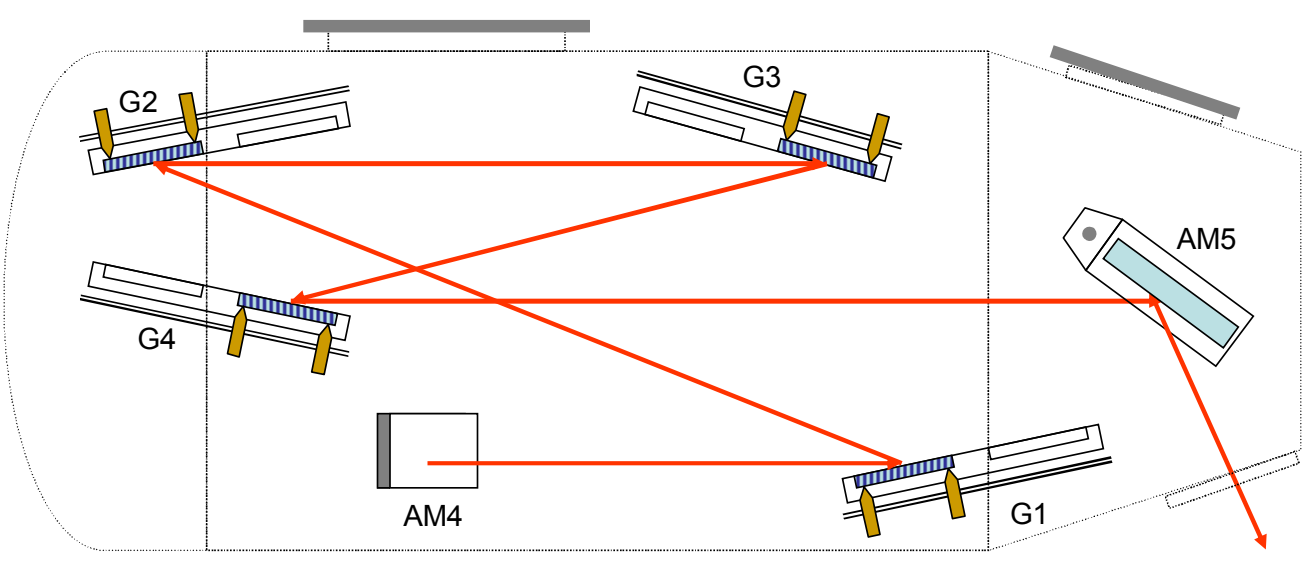

Fig. 2. Compact compressor layout for single beamline.

Research completed under this proposal includes: generating alignment requirements and specifications for the four gratings used in the compact compressor design, defining an offline setup procedure for the gratings using gravity sensors, alignment of the compressors at ambient atmosphere, and reestablishing alignment after vacuum pump down.

\section{Results/Technical Outcome}

The research and development effort proposed for this ER was executed over three years, with the first year primarily focused on developing a detailed set of requirements and specifications that then enabled a useful split beam backlighter system to be implemented. These requirements were then used to develop a detailed modeling and testing capability of the precision metrology and beam pointing system that covers the precision pointing and injection of the chirped pulses into the front end of the amplifier chain and the alignment of the subsequent folded, mixed grating compressor. The work in year two then engaged the experimental demonstration of the split beam technique by demonstrating the precision pointing of the split beams. Also investigated were new techniques for multiple sub-aperture pulse generation, timing and dispersion control. Additionally, diagnostic measurements were performed to demonstrate a suitably low level of cross talk between the split beam apertures in the amplifier system. Finally in year 3, methods for the precision alignment of a folded, mixed grating pulse compressor 
and the effects of vacuum isolation on optical alignment and beam collimation were investigated and characterized.

Detailed requirements and specifications have been generated for split-beam injection into a Nd:Glass laser system. This R\&D effort focused on developing the simplest and most robust approach possible for split beam injection, and differs considerably from approaches that were envisioned only several years ago. Ray tracing and wave optics calculations have been carried out to identify optimal apodizing and spatial shaping masks. Figure 3 shows and example of the spatial shaping masks that were generated during the course of our R\&D. Additionally, R\&D on technology approaches that permit standard nsec-pulse operation as well as short-pulse operation on the same amplifier beam lines with only minor and easily made changes to the beam line have been completed. Importantly, our research has identified an approach in which various components of a single beam line can be shared between a chirped short-pulse as well as a standard nsecs-long pulse simultaneously, permitting the optimum use of all amplifier lines in a system, even when one or several of the amplifier lines are to be used for short-pulse generation.

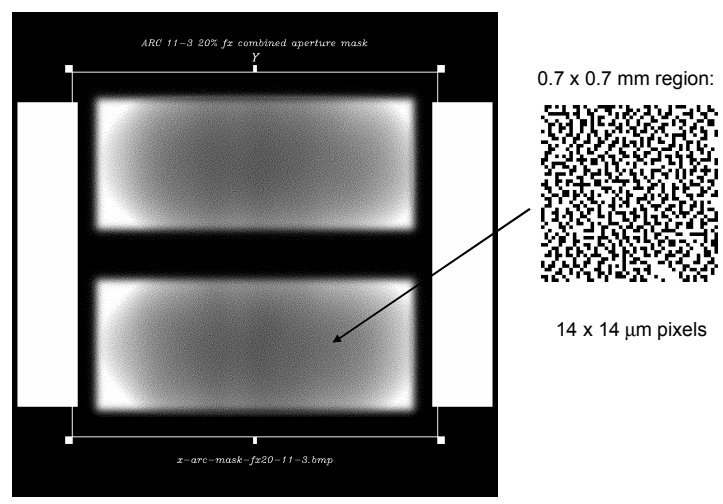

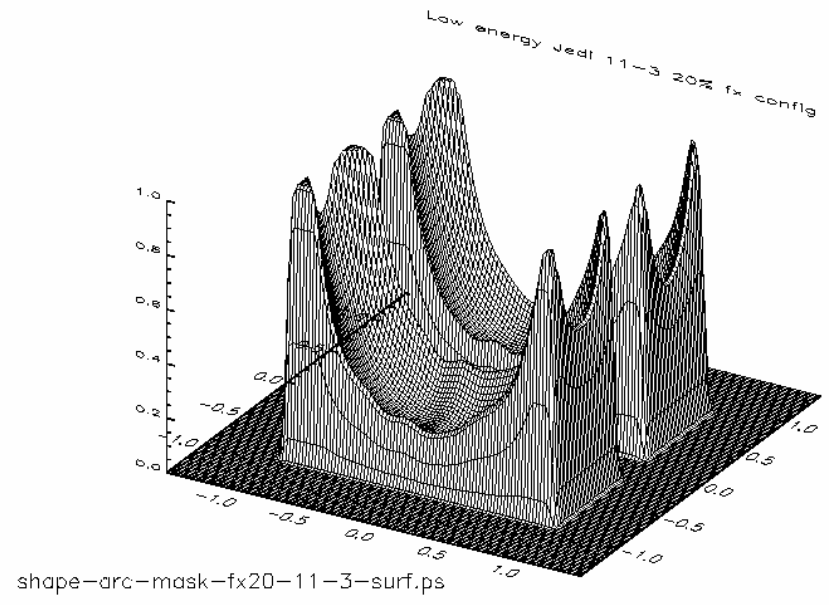

Fig. 3. Shown on the left is the pixilated pattern for the spatial shaping mask for split beam injection that was developed during the course of our R\&D during the last year. On the right is our model prediction for the mask transmission. The very peaked nature of the transmission function is to account for both the gaussian shape of the input split beams, as well as the spatial gain non uniformity in the subsequent amplifier stages.

The ability for this simultaneous use of various beam line components by both chirped short-pulses and a standard nsec-duration pulses was not foreseen at the beginning of this effort, but should greatly enhance the utility of the split beam injection technology being developed in this proposal. Finally, R\&D on diagnostics for the split beam injection has been completed and prototype diagnostic packages have been designed. 
Figure 4 shows a sketch of a an experimental set up, based on R\&D completed during our effort to demonstrate split beam injection. One new feature of this apparatus is enhanced prepulse isolation that is critically important to ensure simultaneous shortpulse and standard nsec-long pulse beam line sharing of various components. This is necessary because the short pulses will generally have to lead the standard nsec-duration pulses in time to account for the additional propagation time required for the compressor at the end of the amplifier chain. Any pulse leakage from the short-pulse into the longpulse beam lines via the shared components could pose an unacceptable prepulse problem for the standard long-pulse beam lines. Figure 5 depicts how we propose to modify a typical beam de-multiplexing system so that it can accommodate simultaneous short pulses along with standard long pulses. .

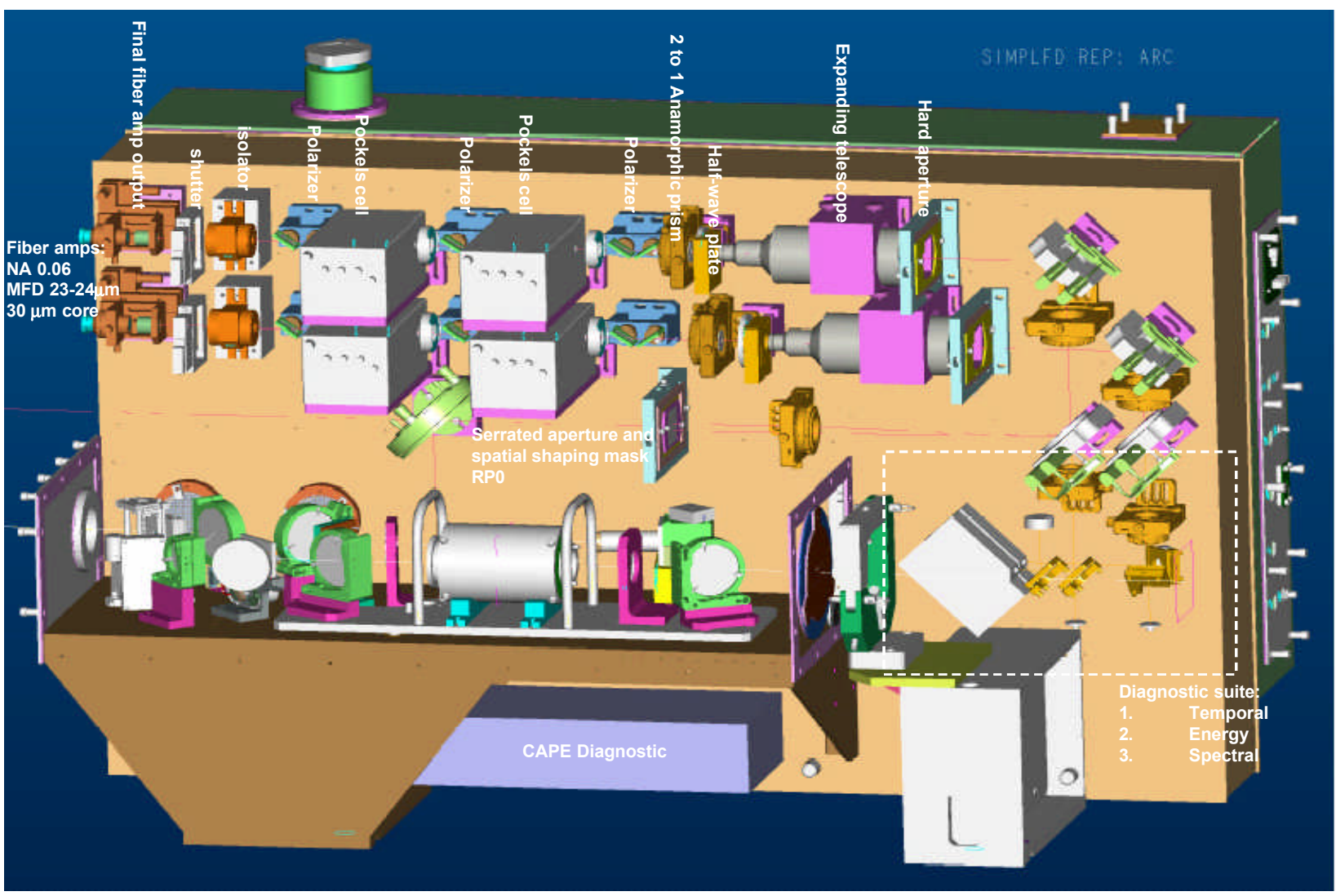

Fig. 4. Experimental setup for evaluating split beam injection. Shown here are the inputs from two independent fiber amplifier outputs (in the upper left hand corner) and the subsequent beam line with diagnostic pickoffs to characterize the split injection beam. 


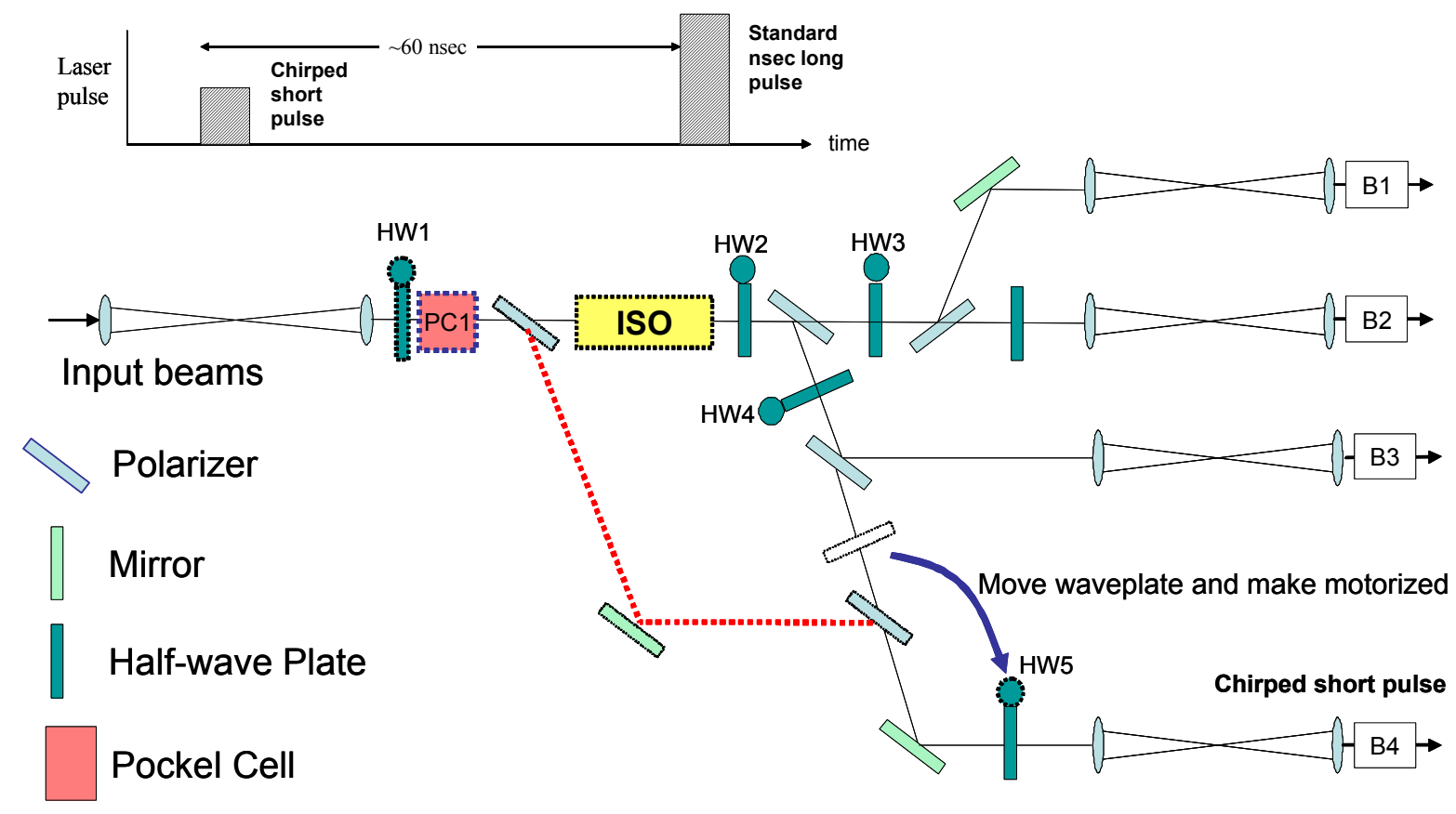

ISO $\mathrm{PC} \gg \mathrm{PC} \&$ Pockels cells and Polarizers will be added for prepulse isolation

Fig. 5. Beam de-multiplexing system in which a single input pulse is split and routed to four different beam lines. Dotted lines depict the changes we are proposing to make, based on R\&D completed during this effort, to best permit simultaneous operation of various beam line components with both chirped shortpulses and standard long pulses. The proposed technique relies on time demultiplexing the input beam line and so requires the use of Pockels cells for time dependent beam line routing.

\section{Summary}

Major advances in short pulse laser technology since the conception of the National Ignition Facility (NIF) have opened up opportunities to deploy powerful new radiographic diagnostics using high energy photons, which should significantly enhance NNSA missions at NIF. The NNSA mission relevance of this ER has been the program relevant science to enable this to occur, and in particular this ER has focused on providing the R\&D base required to successfully implement such an advanced radiographic diagnostic capability. More specifically, the goal of this ER has been to develop the R\&D base to a point that is sufficient to enable the generation of photon energies in the range of $20 \mathrm{keV}$ to $1 \mathrm{Mev}$, using a single amplifier chain or beam line. The benefits of such a capability include 1000 times more penetration through matter and a 10-fold reduction in motion blurring through shorter pulse length than is possible without the introduction of these technological approaches. Within this ER, and to support our goal of developing an advanced radiographic diagnostic capability, we have developed the techniques and technology components that will enable the amplification of two beams simultaneously in a single amplifier chain. This concept for the amplification of split, spatially and temporally independent beams through a single amplifier aperture is novel to LLNL and has not been demonstrated elsewhere. Risk 
issues have been addressed for this proposed technique to be potentially applicable to the generation of multiple time-frame x-ray radiographs of laser driven experiments include: precision parallelism of the input sub-aperture beams and the possibility of cross talk between beams. Results of this ER will are of great interest to the worldwide, high energy, short-pulse community and may become the template for future systems.

\section{References}

1. UCRL-PROP-155637, “Advanced Short Pulse Radiaography diagnostic for NIF, A Science Initiative."

2. IL-1107, "Compact Mixed Grating Optical Pulse Compressor,” C.P. Barty. 